\title{
Effects of mEPO on neurogenesis and gliogenesis after cerebral ischemia in mice
}

\author{
Sijia Zhang, Rongliang Wang, Haiping Zhao, Zhen Tao, Jincheng Li, Fei Ju, Yumin Luo \\ Cerebrovascular Diseases Research Institute, Xuanwu Hospital of Capital Medical University, China
}

Objective: To explore the effects of nonerythropoietic mutant erythropoietin (mEPO) on survival rate, brain loss volume, neurological function, neurogenesis and gliogenesis in mice after middle cerebral artery occlusion. Methods: Forty adult male C57BL/6 mice (25-30g) were randomly divided into sham operation group (sham), ischemia-reperfusion + normal saline control group (I/R+Veh), I/R+EPO group and I/R+mEPO group, with 10 mice in each group. Focal cerebral ischemia was induced by middle cerebral artery occlusion (MCAO). EPO (5000 IU/kg), mEPO (5000 IU/kg) or equal volume of normal saline was injected intraperitoneally every other day after reperfusion. The neurological function of mice was evaluated by rota-rod test at 1, 3, 7 and 14 days after MCAO. Bromodeoxyuridine (BrdU) was injected intraperitoneally daily to analyze the number of newly proliferating cells. The mortality rate of mice, the ratio of brain tissue loss, neurogenesis and gliogenesis were detected 14 days after I/R. Results: there was no significant difference in the loss of brain tissue between $\mathrm{I} / \mathrm{R}+\mathrm{mEPO}$ group and $\mathrm{I} / \mathrm{R}+\mathrm{Veh}$ group $(\mathrm{P}>0.05)$; neurological function and survival rate in $\mathrm{I} / \mathrm{R}+\mathrm{mEPO}$ group had significantly improved compared with $\mathrm{I} / \mathrm{R}+$ Veh group $(\mathrm{P}<0.05)$; the number of $\mathrm{BrdU}+/ \mathrm{NeuN}+$ cells, BrdU+/Dcx+ cells and BrdU+/CNPase in I/R+mEPO group were increased significantly compared with the I/R + Veh group $(\mathrm{P}<0.05)$. In contrast, the number of $\mathrm{BrdU}+/ \mathrm{Iba} 1+$ cells in $\mathrm{I} / \mathrm{R}+\mathrm{mEPO}$ group were significantly reduced than those in $\mathrm{I} / \mathrm{R}+$ Veh group $(\mathrm{P}<0.05)$; among all the tests in this study, there was no significant difference between $\mathrm{I} / \mathrm{R}$ $+\mathrm{mEPO}$ group and $\mathrm{I} / \mathrm{R}+\mathrm{EPO}$ group. Conclusion: $\mathrm{mEPO}$ promoted the regeneration of neurons and oligodendrocytes in peripheral area of cerebral ischemia, and suppressed the proliferation of microglia, thus promoting neurogenesis and improving neurological function and survival rate. Our findings suggest that the nonerythropoietic mutant EPO may be a therapeutic drug for ischemic stroke. 